\title{
Editorial
}

\section{Advances in Computational Imaging: Theory, Algorithms, and Systems}

\author{
Jinyang Liang, ${ }^{1}$ Mohammadreza Nasiriavanaki, ${ }^{2}$ Ziran $W u,{ }^{3}$ and Liang Gao ${ }^{4}$ \\ ${ }^{1}$ Department of Biomedical Engineering, Washington University in St. Louis, St. Louis, MO 63130, USA \\ ${ }^{2}$ Department of Biomedical Engineering, Wayne State University, 818 W. Hancock, Detroit, MI 48201, USA \\ ${ }^{3}$ Physical Optics Corporation, 1845 W 205th St, Torrance, CA 90501, USA \\ ${ }^{4}$ Department of Electrical and Computer Engineering, University of Illinois at Urbana-Champaign, 405 North Mathews Avenue, \\ Urbana, IL 61801, USA \\ Correspondence should be addressed to Jinyang Liang; jinyang.liang@email.wustl.edu
}

Received 21 December 2016; Accepted 28 December 2016; Published 28 February 2017

Copyright (C) 2017 Jinyang Liang et al. This is an open access article distributed under the Creative Commons Attribution License, which permits unrestricted use, distribution, and reproduction in any medium, provided the original work is properly cited.

\section{Introduction}

Computational imaging has emerged as an exciting research area in recent years. Unlike conventional imaging, in which an image is formed directly at the detector, computational imaging inherently integrates computing in the image formation process. In addition, its unique paradigm of data acquisition and image reconstruction marries optical engineering and mathematics. Compared with conventional imaging, this union provides attractive advantages in imaging speed, signal-to-noise ratio, and information throughput. Leveraging novel mathematical models and algorithms with rapidly advancing hardware, computational imaging is versatile in myriad applications, especially where conventional imaging systems are inapplicable.

This special issue is composed of 12 research articles, representing both recent developments in computational imaging algorithms and systems and new applications. A brief description of each paper follows.

\section{Developments in Algorithms and Systems}

In "An Improved Nondominated Sorting Genetic Algorithm for Multiobjective Problem," R. Wang improves the NSGA2 algorithm by adding a local search in each iteration. The new algorithm outperforms the original version in solving two classic multiobjective problems.

A. Sharma et al. present a new algorithm for clusteringIDBSCAN-that is applicable to arbitrary shapes and huge data bases in "Improved Density Based Spatial Clustering of Applications of Noise Clustering Algorithm for Knowledge Discovery in Spatial Data." This algorithm holds promise for a broad range of applications, including medical imaging and remote sensing.

In "Study on MPGA-BP of Gravity Dam Deformation Prediction," X. Wang et al. present a hybrid algorithm that combines a multiple population genetic algorithm (MPGA) and a genetic algorithm-based back-propagation neural network (GA-BP). This new algorithm, which provides superior convergence speed and prediction accuracy, is employed for analyzing the displacement of a dam in China.

J. Zhao et al., in "A Strong Robust Zero-Watermarking Scheme Based on Shearlets' High Ability for Capturing Directional Features," use the Shearlet transform to analyze the direction features in given images. Under various attacks, such as compression, noise addition, and scaling, the proposed watermarking scheme shows superior performance.

In "A Doubly Adaptive Algorithm for Edge Detection in 3D Images" S. Lantaron et al. propose a new algorithm, DA3DED, for edge detection in 3D images. DA3DED is much faster than the conventional $1 \mathrm{D}$ edge detection algorithm for $3 \mathrm{D}$ images.

C. Niyomugabo et al. improve the accuracy of face detection in "A Modified Adaboost Algorithm to Reduce False Positives in Face Detection." The algorithm relies on a new weighting system that gives higher weights to weak classifiers with the best positive classifications. Experimental results show that the new algorithm performs comparably 
to the original Adaboost algorithm but reduces false-positive results by almost four times.

\section{Novel Applications}

In "3D Modeling of Transformer Substation Based on Mapping and 2D Images," L. Sun et al. present a new modeling method with applications in mapping hazardous areas, such as the transformer substation.

Y. Gao et al., in "Restoration and Enhancement of Underwater Images Based on Bright Channel Prior," leverage the bright channel prior and histogram equalization to effectively enhance the quality of underwater images in experiments.

In "Study on Leading Vehicle Detection at Night Based on Multisensor and Image Enhancement Method," M. Chen et al. propose a computational imaging method to detect a vehicle ahead and decrease rear-end accidents. The experimental results show that the method can accurately detect leading vehicles and accurately determine their location.

$Z$. Fu et al. proposed a direction controlled nonlinear least squares (NLS) estimation algorithm using the primal-dual method in "Nonlinear Least Square Based on Control Direction by Dual Method and Its Application." This algorithm shows high precision in analyzing satellite images.

In "A Novel Image Retrieval Based on a Combination of Local and Global Histograms of Visual Words," Z. Mehmood et al. present and demonstrate an effect method for identifying words in various image datasets.

In "3D Reconstruction of End-Effector in Autonomous Positioning Process Using Depth Imaging Device," Y. Hu and L. Li use a simple depth device, assisted by Kalman filtering, a back-propagation neural network, and batch point cloud modeling, to calculate the space coordinates of an endeffector and a target. They fit a 3D surface by using the radial basis function and the morphology. Experimental results demonstrate the effectiveness of this approach in autonomous positioning.

All submitted papers have undergone peer review. We hope that these articles will present exciting developments currently under way and stimulate further advancement in computational imaging.

Jinyang Liang

Mohammadreza Nasiriavanaki

Ziran $W u$

Liang Gao 


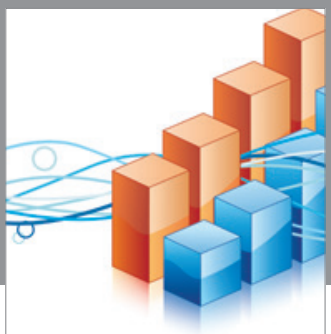

Advances in

Operations Research

vatem alat4

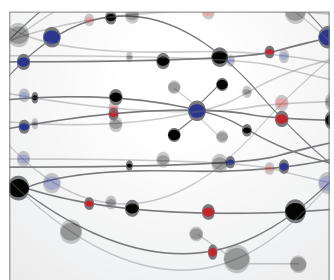

\section{The Scientific} World Journal
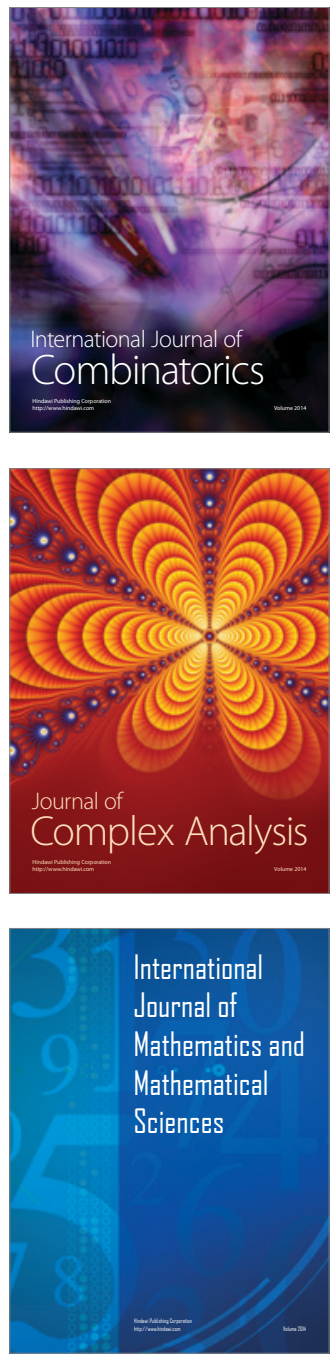
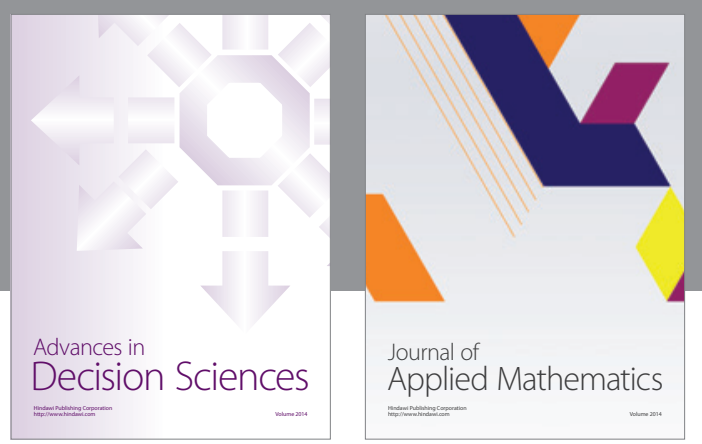

Algebra

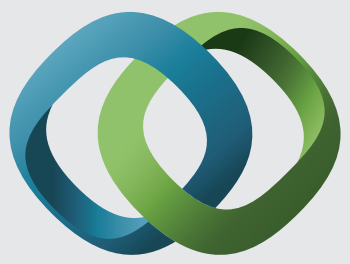

\section{Hindawi}

Submit your manuscripts at

https://www.hindawi.com
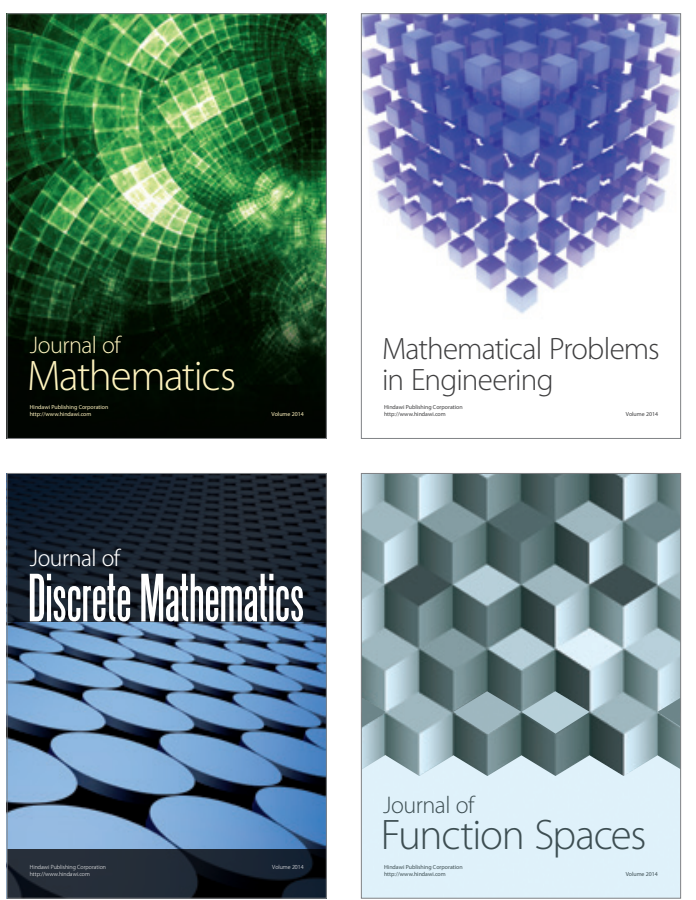

Mathematical Problems in Engineering
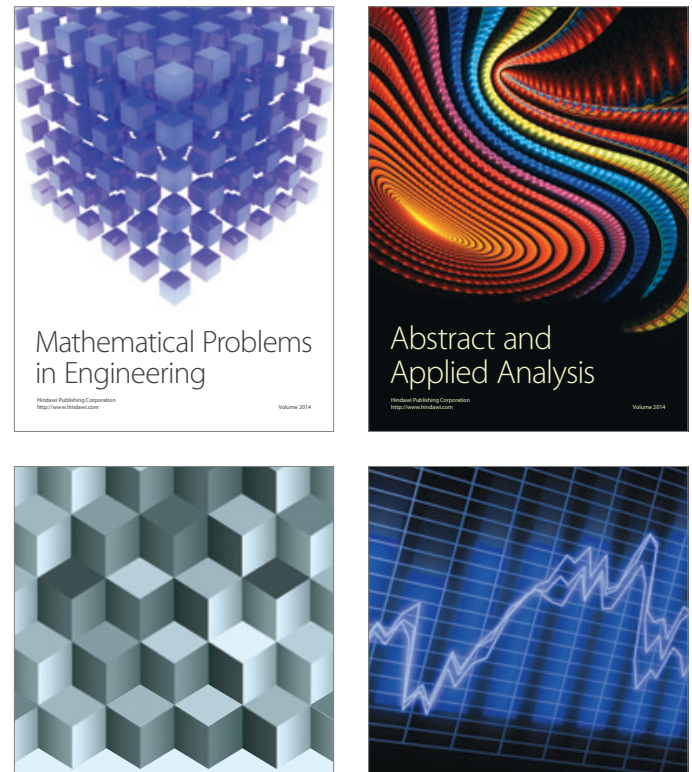

Journal of

Function Spaces

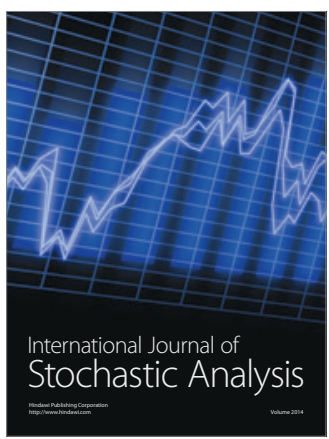

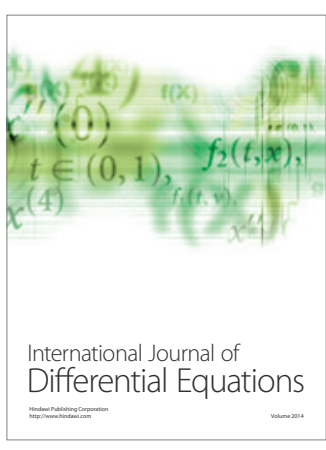
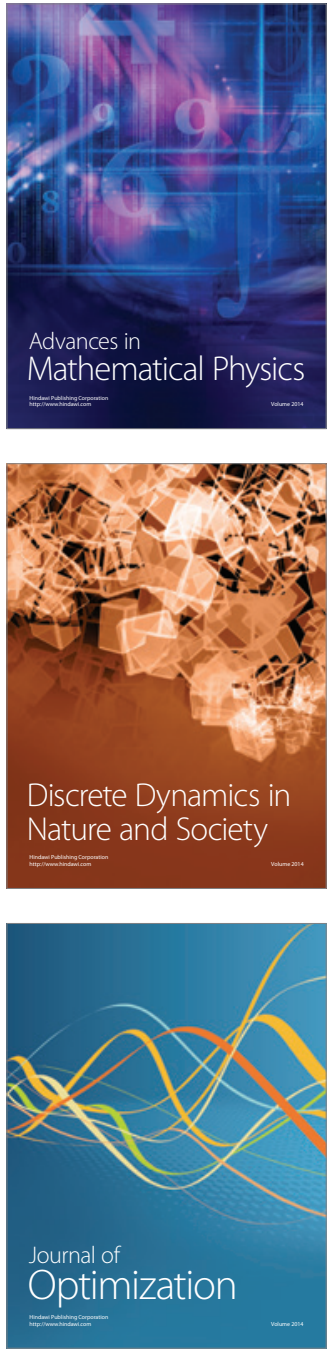